\title{
PD-L1 testing based on the SP142 antibody in metastatic triple-negative breast cancer: summary of an expert round-table discussion
}

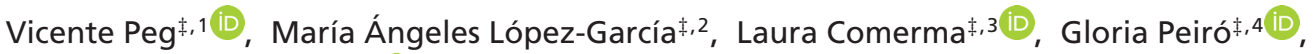 \\ Tomás García-Caballero ${ }^{\ddagger}$, ID $^{\text {, Ángel Concha López }} z^{\ddagger, 6}$, Ana Suárez-Gauthier ${ }^{\ddagger}, 7$, Irune Ruiz $z^{\ddagger, 8}$ \\ \& Federico Rojo $*,+, 9$ (iD) \\ ${ }^{1}$ Departamento de Anatomia Patològica, Vall d'Hebron Hospital Universitari, Barcelona Hospital Campus, Barcelona, Spain; Grupo \\ de Patología Molecular, Vall d'Hebron Institut de Recerca (VHIR), Barcelona, Spain; Centro de Investigación Biomédica en Red en \\ Cáncer (CIBERONC), Instituto de Salud Carlos III, Madrid, Spain; Universitat Autònoma de Barcelona, Bellaterra, Spain \\ ${ }^{2}$ Servicio de Anatomía Patológica, Hospital Universitario Virgen del Rocío, Sevilla (Spain); Centro de Investigacion Biomédica en \\ Red en Cáncer (CIBERONC), Instituto de Salud Carlos III, Madrid, Spain \\ ${ }^{3}$ Departamento de Anatomía Patológica, Hospital del Mar, Barcelona, Spain \\ ${ }^{4}$ Departamento de Patología, Hospital General Universitario de Alicante, Instituto de Investigación Sanitaria y Biomédica de \\ Alicante (ISABIAL), Alicante, Spain \\ ${ }^{5}$ Departamento de Ciencias Morfológicas, Facultad de Medicina y Odontología, Universidad de Santiago, Santiago de \\ Compostela, Spain \\ ${ }^{6}$ Servicio de Anatomía Patológica, Complejo Hospitalario Universitario A Coruña, Biobanco INIBIC, A Coruña, Spain \\ ${ }^{7}$ Departamento de Anatomía Patológica, Laboratorio de Dianas Terapéuticas, Hospital Universitario HM Sanchinarro, Madrid, Spain \\ ${ }^{8}$ Servicio de Patología, Hospital Universitario Donostia, Donostia, Spain \\ ${ }^{9}$ Servicio de Anatomía Patológica, IIS-Fundación Jiménez Díaz-CIBERONC, Madrid, Spain \\ *Author for correspondence: Tel.: +34 915504 800; frojo@fjd.es \\ $\ddagger$ Authors contributed equally
}

Triple-negative breast cancer (TNBC) is more aggressive than other breast cancer subtypes. TNBC is characterized by increased expression of Programmed Death-ligand 1 (PD-L1), a signal used by many tumors to escape the immune response. Expression of PD-L1 is a positive predictor of response to immunotherapy; therefore, it should be investigated in TNBC in order to select patients who may benefit from anti-PD-L1 therapies. While many PD-L1 assays are available, only the VENTANA platform with the anti-PD-L1 (SP142) antibody is licensed as a companion diagnostic device for selecting patients with metastatic/advanced TNBC who are candidates for treatment with atezolizumab. In this article, we provide a summary of an expert round-table discussion about PD-L1 testing, using the SP142 antibody in metastatic TNBC.

Lay abstract: Triple-negative breast cancer (TNBC) is the most aggressive breast cancer subtype. Recent discoveries in TNBC have shown that the higher the expression of the surface molecule PD-L1 in the cancer cells, the better the response of patients to immunotherapy. While several tests or diagnostics assays for detecting PD-L1 exist, only the antibody anti-PD-L1 SP142 possesses proven diagnostic value for selecting metastatic TNBC patients eligible for atezolizumab immunotherapy. Throughout the present article, a group of experts discusses how to best carry out the assessment of PD-L1 status with this assay.

First draft submitted: 29 October 2020; Accepted for publication: 19 November 2020; Published online: 8 December 2020

Keywords: antibody $\bullet$ breast cancer $\bullet$ diagnosis $\bullet$ immunohistochemistry $\bullet$ immunotherapy $\bullet$ PD-L1

Advances in our knowledge of the oncogenesis of breast cancer and the need to improve prognosis have led to the development of new treatments to target molecules involved in the growth and proliferation of tumor cells (TCs). However, in recent years, new immunotherapies have been designed to treat breast tumors [1].

Many TCs can escape the immune system using various mechanisms, such as mutation or loss of tumor-specific antigens, loss of MHC class I protein expression, defects in antigen processing and presentation pathways, T-cell receptor signaling and costimulation, upregulation of inhibitory immune checkpoint pathways by TCs and/or 
immune cells (ICs) within the tumor microenvironment, namely, the PD-1 axis [2]. When PD-1 expressed on ICs is activated by its ligands, PD-L1 or PD-L2, it attenuates lymphocyte activation and promotes the development of functional Treg, thereby enabling inhibition of the immune response [2,3]. As many tumors use this strategy to escape the immune system, the PD-1 signaling pathway has become an interesting therapeutic target in cancer [4].

Triple-negative breast cancer (TNBC) tends to be more aggressive than other breast cancer subtypes. In fact, until very recently, the only available approach to date was cytotoxic chemotherapy [5-9]. However, experts in this field are witnessing with hope recent approvals for molecularly targeted therapies: two PARP inhibitors (olaparib and talazoparib) for germline BRCA-mutated breast cancers and two checkpoint inhibitors, in other words, atezolizumab in combination with nab-paclitaxel for (PD-L1+) and pembrolizumab in the neoadjuvant setting for advanced TNBC [10]. Interestingly, TNBC has proven to be more immunogenic than other breast cancer subtypes, as evidenced by the marked presence of tumor-infiltrating lymphocytes within the tumor microenvironment $[10,11]$ and overexpression of PD-L1 mRNA [12]. Therefore, the PD-L1/PD-1 axis has emerged as an exciting therapeutic target in this subtype of breast cancer [13].

Upregulation of PD-L1 in locally advanced or metastatic TNBC (mTNBC) has proven to be associated with better survival in patients treated with checkpoint inhibitors. A recent study that assessed the safety and clinical activity of atezolizumab in TNBC showed that patients with PD-L1 expression of at least $1 \%$ tumor-infiltrating ICs had a higher objective response rate (ORR) (12\%) and longer overall survival (OS; 10.1 months) than those with less than 1\% ICs (ORR 0\% and OS 6.0 months) [14]. This finding was confirmed in the Phase III IMpassion130 trial, where a clinically significant improvement in OS of 7.5 months (hazard ratio [HR]: 0.67; 95\% CI: 0.530.86; not formally tested due to the hierarchical study design) was observed in the PD-L1-positive population [15]. Similarly, in a Phase Ib study testing single-agent pembrolizumab in TNBC, the ORR was approximately $19 \%$ in PD-L1-selected patients, defined by a combined positive score (CPS) of at least $1 \%$ with the $22 \mathrm{C} 3$ scoring system (CPS is defined as the number of PD-L1 staining cells [TCs, lymphocytes, macrophages] divided by the total viable TCs, multiplied by 100) [16]. Likewise, in the Phase III KEYNOTE-355 study, progression-free survival (PFS) improved in the PD-L1-positive population (HR: 0.65; 95\% CI: 0.49-0.86; $\mathrm{p}=0.0012$ ), although no OS data were presented [17]. All of these results highlight the importance of assessing PD-L1 expression levels in TNBC to select patients who will derive greater benefit from these therapies.

In this article, we present the opinion of a group of experts on how to assess PD-L1 status using SP142 in patients with $\mathrm{mTNBC}$ in clinical practice.

\section{Methods for assessing PD-L1 expression}

Several PD-L1 assays are used to select patients for immunotherapy. These assays have been developed independently for specific drugs - based on different antibody clones, staining protocols and platforms, and scoring algorithms and define the cut-off points for PD-L1-positive or -negative expression on TCs or on ICs [18]. Since each antibody clone recognizes a specific epitope of PD-L1, its specific staining pattern, while similar, is not fully concordant with the others [19-22].

The most common commercially available monoclonal PD-L1 antibodies for immunohistochemical analysis to assess the expression of PD-L1 are the following: 22C3, 28-8, SP142, SP263 and 73-10 (Table 1) [19,23,24]. In metastatic non-small-cell lung cancer, the 22C 3 antibody clone is used as a companion assay to select patients for pembrolizumab monotherapy, with a tumor proportion score (TPS) $\geq 1 \%$ being the cut-off for first-line treatment. The 28-8 clone is used as a complementary assay for second-line treatment with nivolumab, with a TC $\geq 1 \%$ cut-off. The SP142 antibody is used as a complementary assay for second-line treatment with atezolizumab, with a cut-off of $\geq 1 \%$ of TCs and $\geq 1 \%$ of ICs. More importantly, it is classified by the US FDA as a companion diagnostic for first-line atezolizumab monotherapy, assessed based on a TC $\geq 50 \%$ and IC $\geq 10 \%$ cut-off. Finally, the SP263 antibody can be used as a companion or complementary assay for approved immuno-oncology therapies $[19,25,26]$.

Each antibody clone uses its own immunohistochemistry assay staining protocol. 22C3, 28-8 and 73-10 use the DAKO Autostainer Link 48 platform (Agilent Inc., CA, USA) and SP142 and SP263 use the VENTANA BenchMark Ultra platform (Ventana Medical Systems Inc., AZ, USA) (Table 1) [19]. The use of one or another detection method (platform and immunohistochemistry reagents) can influence the identification of PD-L1positive TCs or ICs, thus indicating the significant role of the detection method, even for the PD-L1 antibody clone [19]. Moreover, each assay has a different sensitivity for detection of PD-L1 depending on whether the test is aimed at detecting TCs or ICs. For example, with the Lab Vision Autostainer $480 \mathrm{~S}$ platform (Thermo Fisher Scientific, MA, USA), SP142 stained more TCs than usual when applied as per the method sheet and, therefore, 
Table 1. Most common commercially available monoclonal PD-L1 antibodies for immunohistochemical analysis to assess the expression of PD-L1 considering US FDA approvals for non-small-cell lung cancer.

\begin{tabular}{|c|c|c|c|c|c|}
\hline PD-L1 antibody & Drug & IHC assay & Cut-off & Line & Approval status \\
\hline \multirow[t]{2}{*}{$22 \mathrm{C} 3$} & \multirow[t]{2}{*}{ Pembrolizumab } & \multirow[t]{2}{*}{$\begin{array}{l}\text { DAKO Autostainer Link } 48 \text { platform } \\
\text { (Agilent Inc) }\end{array}$} & \multirow[t]{2}{*}{ TPS $\geq 1 \%$} & \multirow[t]{2}{*}{$1 \mathrm{~L} 2 \mathrm{~L}$} & $\begin{array}{l}\text { - FDA: companion } \\
\text { - CE-IVD }\end{array}$ \\
\hline & & & & & $\begin{array}{l}\text { - FDA: companion } \\
\text { - CE-IVD }\end{array}$ \\
\hline $28-8$ & Nivolumab & $\begin{array}{l}\text { DAKO Autostainer Link } 48 \text { platform } \\
\text { (Agilent Inc) }\end{array}$ & $\mathrm{TC} \geq 1 \%$ & $2 \mathrm{~L}$ & $\begin{array}{l}\text { - FDA: complementary } \\
\text { - CE-IVD }\end{array}$ \\
\hline \multirow[t]{2}{*}{ SP142 } & \multirow[t]{2}{*}{ Atezolizumab } & \multirow[t]{2}{*}{$\begin{array}{l}\text { VENTANA BenchMark Ultra platform } \\
\text { (Ventana Medical Systems Inc) }\end{array}$} & $\begin{array}{l}\mathrm{TC} \geq 50 \% \text { and } / \text { or } \mathrm{IC} \\
\geq 10 \%\end{array}$ & $1 \mathrm{~L}$ & $\begin{array}{l}\text { - FDA: companion } \\
\text { - CE-IVD }\end{array}$ \\
\hline & & & $\begin{array}{l}\mathrm{TC} \geq 1 \% \text { and } / \text { or IC } \\
\geq 1 \%\end{array}$ & $2 \mathrm{~L}$ & $\begin{array}{l}\text { - FDA: complementary } \\
\text { - CE-IVD }\end{array}$ \\
\hline \multirow[t]{3}{*}{ SP263 } & Durvalumab & \multirow[t]{3}{*}{$\begin{array}{l}\text { VENTANA BenchMark Ultra platform } \\
\text { (Ventana Medical Systems Inc) }\end{array}$} & $\mathrm{TC} \geq 1 \%$ & $\begin{array}{l}1 \mathrm{~L} \text { maintenance, in } \\
\text { unresectable stage III after } \\
\text { chemoradiation therapy }\end{array}$ & $\begin{array}{l}\text { - FDA: } \\
\text { complementary/companion } \\
\text { - CE-IVD: companion }\end{array}$ \\
\hline & Nivolumab & & $\mathrm{TC} \geq 1 \%$ & $2 \mathrm{~L}$ & - CE-IVD \\
\hline & Pembrolizumab & & $\mathrm{TC} \geq 50 \%$ & $1 \mathrm{~L}$ & - CE-IVD \\
\hline $73-10$ & Avelumab & $\begin{array}{l}\text { DAKO Autostainer Link } 48 \text { platform } \\
\text { (Agilent Inc) }\end{array}$ & $\mathrm{TC} \geq 1 \%$ & $2 \mathrm{~L}$ & - NA \\
\hline $\begin{array}{l}\text { CE-IVD: European } \\
\text { Second line. } \\
\text { Reproduced from }\end{array}$ & mmission in vitr & ; IC: Immune cell; IHC: Immunohistoc & & & Not approved; 1L: First line; \\
\hline
\end{tabular}

was more concordant with the $22 \mathrm{C} 3$ and $28-8$ kits [19]. Thus, the staining pattern of the SP142 assay - and not only the antibody - reveals more ICs but fewer TCs than the other PD-L1 assays.

The 22C3 and 28-8 assays, on the other hand, are highly sensitive for TCs, although their staining for ICs is less pronounced [19]. Questions have therefore been raised about whether the tests can be used interchangeably to inform treatment decisions for the various PD-1 and PD-L1 inhibitors.

Although the DAKO and VENTANA platforms are both used in clinical practice, a companion diagnostic label involves a whole procedure [27]. Thus, using SP142 as a companion diagnostic for nab-paclitaxel + atezolizumab in mTNBC involves the whole procedure based on the PD-L1 (SP142) antibody performed in the VENTANA platform and following the protocol provided by the vendor [28]. Importantly, the FDA approval of the VENTANA platform as a companion diagnostic for atezolizumab in TNBC was based on IMpassion130, a multicenter, international, double-blinded, placebo-controlled, randomized trial that assessed the efficacy and safety of atezolizumab plus nab-paclitaxel in patients with unresectable, locally advanced or mTNBC $[29,30]$.

\section{Discussion}

At the end of 2019, an expert meeting was held to discuss the various methods for assessing expression of PD-L1 in patients with mTNBC. Special emphasis was given to the VENTANA platform and the SP142 antibody clone as the only option with proven clinical utility for the selection of patients with mTNBC who were candidates for treatment with atezolizumab. The questions raised at the meeting are addressed below.

\section{Given the available clinical evidence, is SP142 currently the reference diagnostic method for} assessing PD-L1 status in MTNBC?

The VENTANA PD-L1 (SP142) assay has proven to be highly sensitive for ICs and less sensitive for TCs [31], whereas the DAKO 22C3 and 28-8 assays show similar sensitivity for PD-L1 expression on TCs and lower sensitivity for PD-L1 staining on ICs [19]. Nevertheless, several studies have shown the benefit of using the VENTANA PD-L1 (SP142) assay to identify which patients with mTNBC will more likely benefit from treatment with atezolizumab [29,30,32].

According to the IMpassion 130 trial, detection of PD-L1-positive ICs by VENTANA PD-L1 (SP142) predicted PFS and OS benefits with atezolizumab plus nab-paclitaxel in mTNBC patients $[15,29,30]$. This trial assessed PDL1 expression on ICs using the VENTANA PD-L1 (SP142) immunohistochemistry assay with a $\geq 1 \%$ cut-off for positivity. In the PD-L1-positive group, the PFS was 7.5 months in patients treated with atezolizumab plus nab-paclitaxel and 5.0 months in patients treated with nab-paclitaxel (HR: 0.62; 95\% CI: 0.49-0.78; p < 0.001). 
Similarly, OS was 25.4 months in PD-L1-positive patients treated with atezolizumab plus nab-paclitaxel and 17.9 months in patients receiving nab-paclitaxel (not formally tested due to the prespecified hierarchical statistical plan) $[15,29,30]$. The study concluded that the combination of atezolizumab plus nab-paclitaxel provides a clinical benefit, especially in the PD-L1-positive subgroup. Therefore, it is crucial to assess PD-L1 expression on ICs to inform treatment choices for mTNBC patients [29,30].

As explained in detail above, PD-L1 expression can be evaluated using various immunohistochemistry assays. The post-hoc analysis of IMpassion130 assessed analytical and clinical concordance for the DAKO 22C3, VENTANA SP142 and VENTANA SP263 assays [32]. In this analysis, two algorithms were run to evaluate PD-L1. The VENTANA SP142 and VENTANA SP263 assays followed a PD-L1 IC algorithm, defined as the presence of discernible PD-L1 staining of any intensity in ICs covering $\geq 1 \%$ of tumor area occupied by TCs and associated intratumoral and continuous peritumoral stroma. The DAKO 22C3, on the other hand, followed a CPS algorithm, understood as the number of PD-L1-stained cells (TCs, lymphocytes and macrophages) divided by the total number of viable TCs, multiplied by 100 . According to the results of this study, the immunohistochemistry assays were not interchangeable. The overall percentage agreement was 69\% between VENTANA SP142 and SP263 and 64\% between VENTANA SP142 and DAKO 22C3 [32]. In addition, the results showed that the clinical benefit in the subgroups that were positive for DAKO 22C3 (HR: 0.78; 95\% CI: 0.62-0.99) and VENTANA SP263 (HR: 0.75; 95\% CI: 0.59-0.96) was driven by the VENTANA SP142-positive subgroup (HR: 0.71; 95\% CI: 0.52-0.98). Apart from the benefit observed in terms of HR: SP142-positive subgroups also had the longest median values for PFS (8.3 vs 3.9 months) and OS (27.3 vs 17.9 months) [32]. These results led to the VENTANA PD-L1 (SP142) assay at an IC cut-off $\geq 1 \%$ becoming the approved diagnostic test for identification of mTNBC patients most likely to benefit from the addition of atezolizumab to nab-paclitaxel.

Additionally, according to recent data from the KEYNOTE-355 trial, the combination of pembrolizumab and several chemotherapy backbones produced a statistically significant and clinically meaningful improvement in PFS in patients with previously untreated locally recurrent inoperable TNBC or mTNBC whose tumors expressed PD-L1 (CPS $\geq 1$ or $10 \%$ ), as assessed using the $22 \mathrm{C} 3$ pharmDx assay. In the CPS $\geq 10 \%$ population, the median PFS of the combination was 9.7 months, and the median PFS of chemotherapy alone was 5.6 months (HR: 0.65; 95\% CI: 0.49-0.86) [17]. Nevertheless, no data for OS were presented after 25.9 months of follow-up. These results might lead to the approval of pembrolizumab for PD-L1 (22C3)-selected mTNBC patients, since this drug is not currently approved as first-line treatment in these patients.

\section{Is interpretation based on SP142 reproducible?}

Between March and September 2019, 83 pathologists from various hospitals in Spain participated in a TNBC training program for the VENTANA PD-L1 (SP142) assay. Each session involved a theoretical and a practical component. Pathologists were trained to identify the presence or absence of discernible PD-L1 staining of any intensity in ICs covering a certain percentage of tumor area occupied by TCs and associated intratumoral and adjacent peri-tumoral stroma and, eventually, assigned each case a binary score when $<1 \%$ IC or $\geq 1 \%$ IC, as described in the VENTANA PD-L1 (SP142) assay interpretation guide for TNBC [33]. Therefore, a proficiency test on 28 digital whole scanned cases produced two results for each case and each participating pathologist: one raw score (IC \%) and one binary result: positive or negative. The result for each assessed case was compared with a consensus score established by an expert panel with prior established experience in PD-L1 scoring. The interobserver concordance analysis revealed a Spearman rank correlation coefficient of $0.970(\mathrm{p}<0.001)$, indicating a good correlation between the participants' observations and the consensus scores previously established by the expert panel. Figure 1 shows all numerical observations made by the participants.

These results are similar to those reported elsewhere [23,34,35]. Several trials have assessed the inter-observer reproducibility of the results of the VENTANA PD-1 (SP142) assay. Noske et al. performed the first multicenter study based on staining of whole slides in TNBC with trained readers in a real-world setting to compare the reproducibility and analytical concordance of PD-L1 IC positivity between four anti-PD-L1 assays: VENTANA SP263, VENTANA SP142, DAKO 22C3 and DAKO 28-8 [22]. Importantly, inter-reader agreement on PD-L1 IC positivity was higher with VENTANA SP142 than VENTANA SP263, DAKO 22C3 and DAKO 28-8 [22]. This high inter-reader agreement was confirmed in another study, which reported the results of a global training program for the VENTANA SP142 assay with respect to interpretation of PD-L1 expression in TNBC [34]. However, assessment of interobserver concordance using other methods has not yielded the same results. Reisenbichler $e t a l$. concluded that IC scoring with both the SP142 and the SP263 assays showed poor reproducibility across multiple 


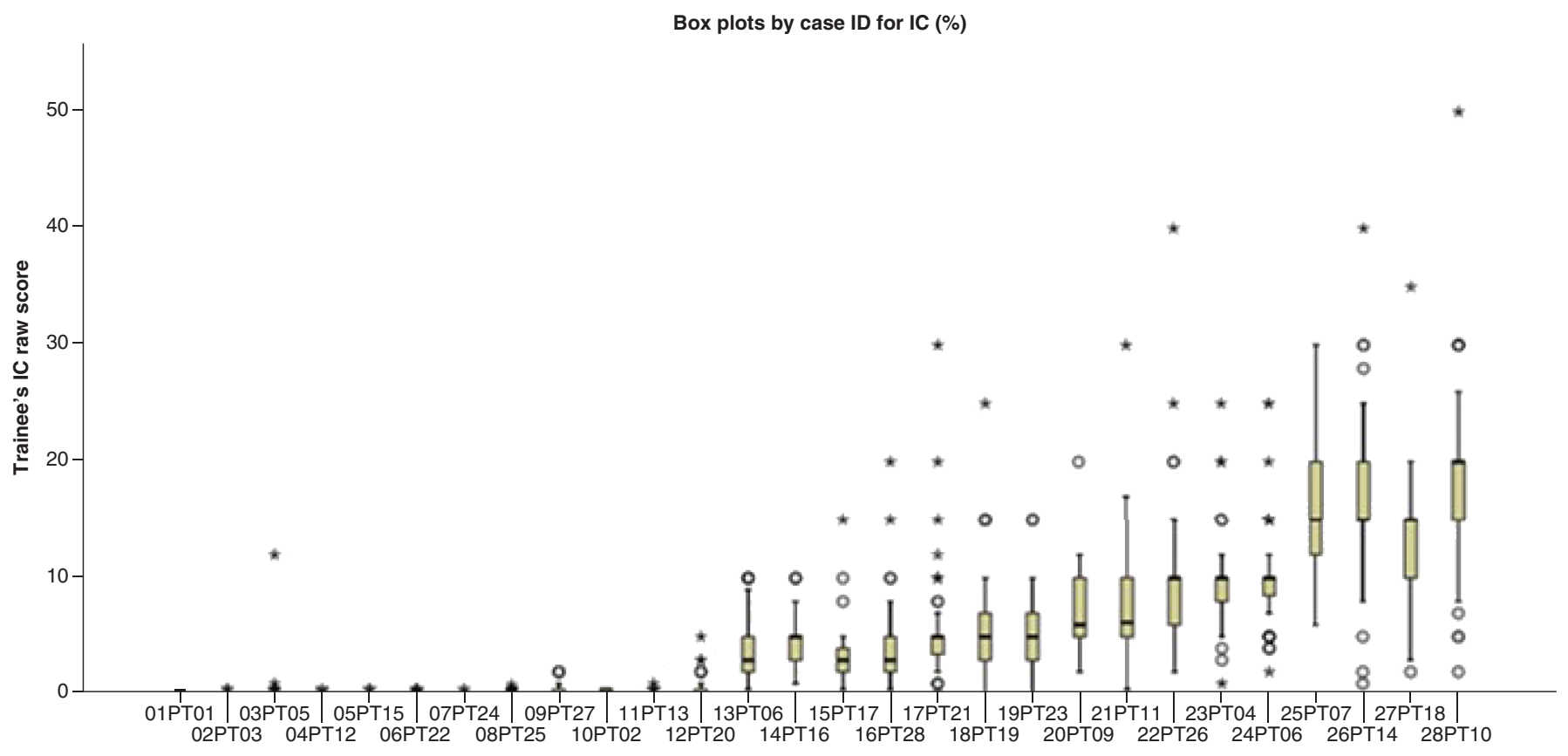

Figure 1. Analysis of agreement between pathologists in the Spanish PD-L1 (SP142) triple-negative breast cancer training program. Box plot by case ID for immune cell percentage. PD-L1 (SP142) assay triple-negative breast cancer mean proficiency trainee test scores compared with expert consensus scores and case-level agreement. Scores for each case were averaged across a total of 83 pathologists who attended the training program. The median for each dataset is indicated by the black center line, and the first and third quartiles are the edges of the box area, which is known as the IQR. The extreme values (within 1.5-times the IQR from the upper or lower quartile) are the ends of the lines extending from the IQR (circles). Points at a greater distance from the median than 1.5-times the IQR are plotted individually as asterisks. These points represent potential outliers.

IC: Immune cell; IQR: Interquartile range.

pathologists using their Observers Needed to Evaluate Subjective Tests analysis. The authors suggest that an assay similar to this one should be interpreted by at least eight to ten pathologists rather than two or three in order to provide a true picture of the reproducibility of the assay in a real-world setting [35].

What is the recommended PD-L1 diagnostic algorithm? Should this biomarker be included in parallel to HER2 \& hormone receptor testing?

In general terms, it was concluded that PD-L1 should be determined in all mTNBC patients. Thus, once a negative result has been confirmed for HER2, estrogen and progesterone receptors, thus leading to a diagnosis of TNBC, PD-L1 should be performed in parallel. However, divergences emerged as to whether PD-L1 determination should be done in the early stage of the disease or only when the tumor has metastasized. According to the clinical course of TNBC, most patients presenting with metastases will experience recurrences from early stages (94\%), whereas those with de novo metastases will be a minority (6\%) [36]. Therefore, PD-L1 could be assessed in early TNBC or once the disease has metastasized. Some experts believed that considering the bad prognosis and the pressing unmet needs of TNBC patients, it would be beneficial to perform the PD-L1 evaluation in early-stage TNBC so that the PD-L1 status was known before the tumor metastasized. In contrast, other experts thought that considering that not all early-stage TNBC recurs, PD-L1 should be assessed once the disease has metastasized in order to ensure a sustainable use of clinical resources. Moreover, considering emerging data from clinical research in TNBC, the therapeutic options would need to be further evaluated using biomarkers upon recurrence. Therefore, no consensus was achieved on whether the evaluation should be performed in early-stage TNBC or mTNBC.

How does the frequency of PD-L1 differ depending on the sample evaluated? Should any tissue be prioritized? How many tests are needed to define a patient's PD-L1 status?

According to various studies, expression of PD-L1 differs significantly between primary and metastatic tumors and is more frequent in primary tumors [37,38]. In their post-hoc analysis of IMpassion130 samples, Rugo et al. reported that expression of PD-L1 in primary tissue was higher than in metastasis ( $44 \mathrm{vs} 36 \% ; \mathrm{p}=0.014$ ). They also showed 


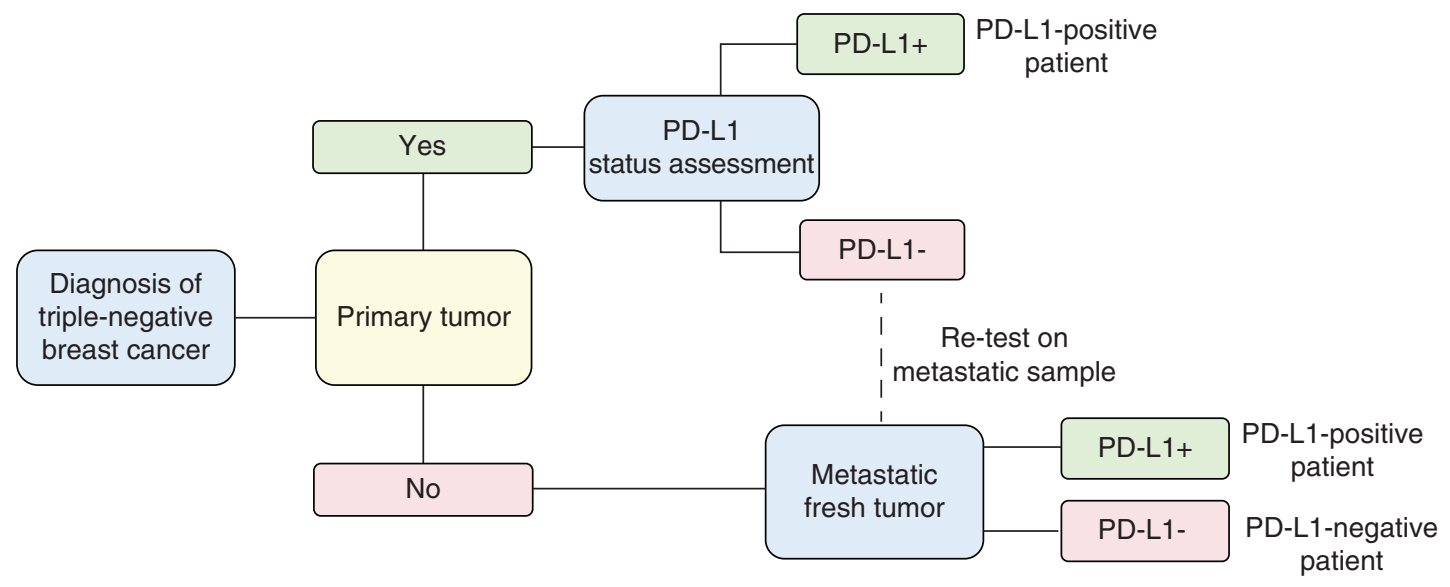

Figure 2. Proposed PD-L1 testing algorithm in metastatic triple-negative breast cancer.

that PD-L1 remains a predictor of response, regardless of the origin of the sample [32]. Therefore, primary tissue should be prioritized when addressing PD-L1 expression in mTNBC patients. Nevertheless, not all primary tumors are appropriate. Data on PD-L1 expression in residual invasive cancer after neoadjuvant chemotherapy are not consistent. Some authors report lower PD-L1 values in the residual tumor than in the primary carcinoma [39,40], although contrasting findings were recently published [41]. Given the scarcity of available data and the discrepancies observed, the expert committee recommends avoiding these samples when analyzing PD-L1, unless no other sample is available.

Few data have been reported on concordance of PD-L1 in matched specimens; however, available evidence suggests that results may be discordant in a percentage of patients [37]. Therefore, if PD-L1 is evaluated only in the primary tumor, PD-L1 status may be misclassified, since, according to the IMpassion 130 trial design, if multiple tumor specimens are submitted for enrollment, the PD-L1 score used to stratify the patient is the maximum score. Taking into account the above-mentioned observations, the expert committee recommends evaluating the expression of PD-L1 first in the primary tumor and, if the result is negative, at least in one metastasis. The proposed workflow is shown in Figure 2.

Importantly, since mTNBC patients have a poor prognosis, therapy must be chosen as soon as possible. At this meeting, the time that a TNBC patient can wait for a biomarker-based diagnosis was not discussed; therefore, it is essential to consider this aspect in future consensus discussions.

Should an order of priority be established according to the origin of the metastatic tissue sample? As stated previously and shown in Figure 2, the primary tumor is the priority sample for evaluation of PD-L1; metastases should only be tested if the primary tumor is negative or not suitable.

Expression of PD-L1 differs according to anatomical location; expression of PD-L1 in ICs is more frequent in lymph nodes (51\%), followed by skin (48\%), lungs (43\%), breast (43\%) and other locations (36\%) [32]. Yuan et al. reported significant differences in expression of PD-L1 between primary breast tumors and paired axillary lymph nodes. The positive rate of PD-L1 expression in primary tumors and matched lymph nodes was 29.8 and $14.8 \%$, respectively $(\mathrm{p}=0.016)$ [42]. These authors also observed that positivity of PD-L1 in metastatic lymph nodes was significantly associated with poor prognostic features, such as high Ki-67 index, high TNM stage, high histology grade and large number of metastatic lymph nodes [42].

Taking the above into account, priority should be given according to positivity; consequently, lymph nodes are the sample of choice for evaluation of PD-L1 in metastases.

When assessing the expression of PD-L1 in metastatic samples, should we take any specific aspects into account (e.g., are there any samples we should avoid)?

Bone samples are not recommended for assessing the expression of PD-L1, as aggressive decalcification treatment decreases the antigenicity of the samples [43]. In addition, assessment of PD-L1 in liver metastases should be avoided, given that this sample type is rarely associated with tumor infiltrating lymphocytes or an immunosuppressive environment [44]. However, there are no published data advising against these samples, since they may be the only 
available metastatic samples, and any positive sample is sufficient to identify atezolizumab-eligible patients. Thus, the recommendation is that these samples should not be prioritized or be the first to be tested.

Furthermore, lymph node metastases are complex to evaluate for various reasons. First, the VENTANA PD-L1 (SP142) assay considers only ICs [45]. Second, lymph nodes are rich in ICs. Third, it is difficult to delimit the tumor area and tumor stroma in lymphoid tissue. Therefore, the expert panel consulted here concluded that, given the high frequency of PD-L1 positivity and the distinctiveness of lymph node metastases, special care is required with this sample. In cases of doubt, an expert should be consulted.

Given the current immunotherapy landscape \& the large number of ongoing clinical trials, is it useful to specify the antibody clone used for assessment of PD-L1 status in the pathology report?

Because several approved immunotherapy options target the PD-L1/PD-1 axis in various types of cancer, it is always necessary to specify the antibody clone used in the assessment of this biomarker in the pathology report. As previously mentioned, each antibody clone has been validated concomitantly with the clinical development of antiPD-1/PD-L1 therapy $[18,19,23]$. Therefore, in the report for TNBC, it is necessary to specify not only the PD-L1 antibody clone assayed (22C3, 28-8, SP142, SP263 and 73-10), but also the platform used for its detection (DAKO Autostainer Link 48 platform or VENTANA BenchMark Ultra platform). In addition, guidelines recommend that both the qualitative value of the expression (positive vs negative) and the quantitative value (percentage of positive TCs and percentage of positive ICs) be specified [46]. This approach is important when deciding on the best treatment from among newly introduced therapeutic agents in clinical practice. Furthermore, and especially in the case of the SP142 clone, it is strongly recommended to report the proportion of the tumor area occupied by ICs [47].

\section{Future perspective}

Immunotherapy is increasingly used in many tumors because of the significant clinical advantages it provides. However, not all patients benefit from it. To select the best candidates for immunotherapy, it is necessary to search for and identify biomarkers with a strong predictive value that are expressed by specific patient populations. This has proved to be the case for PD-L1 in mTNBC. Detection of PD-L1 in these tumors makes it possible to prescribe anti-PD-L1 treatment such as atezolizumab and thus achieve an optimal response.

However, in addition to enabling detection of this biomarker, the procedure (i.e., immunohistochemistry staining followed by scoring) needs to be effective and reproducible to guarantee that the selected therapy is appropriate for the individual patient. Although many advances have been made in this regard, further concordance studies, protocols, and guidelines are still needed to help physicians determine which samples to use, at which stage of the disease to test and, in summary, how to detect PD-L1 more consistently for the benefit of the patient.

Financial \& competing interests disclosure

This article was funded by Roche Diagnostics S.L. The present work was supported by grants from the Spanish Ministry of Economy and Competitiveness (MINECO) with European Regional Development Fund (ERDF) funding through the Institute of Health Carlos III (AES Program, PI15/00934; CIBERONC, Biomedical Research Networking Centre for Cancer). V Peg has received grants from Roche; held advisory roles for Roche, MSD and AstraZeneca and has received honoraria from Sysmex Spain. MA López has no conflict of interests. L Comerma reports being advisory board member and receiving other honoraria as speaker's bureau from Roche. I Ruiz reports being member of expert panels and lectures for Roche and AstraZeneca. F Rojo reports being advisory board member for Roche, MSD, Bristol-Myers Squibb, AstraZeneca, Novartis, Pfizer, Abbvie, InCyte and Pierre Fabre. The authors have no other relevant affiliations or financial involvement with any organization or entity with a financial interest in or financial conflict with the subject matter or materials discussed in the manuscript apart from those disclosed.

The authors wish to thank Content Ed Net (Madrid) and Fernando Sánchez Barbero PhD for the support in the preparation of this manuscript. Writing assistance was funded by Roche Diagnostics S.L.

\section{Open access}

This work is licensed under the Attribution-NonCommercial-NoDerivatives 4.0 Unported License. To view a copy of this license, visit http://creativecommons.org/licenses/by-nc-nd/4.0/ 
Executive summary

Introduction

- Triple-negative breast cancer (TNBC) tends to be more aggressive than other breast cancer subtypes.

- TNBC has proven to be more immunogenic than other breast cancer subtypes, with higher expression of PD-L1.

- PD-1/PD-L1 has emerged as a promising therapeutic target in breast cancer subtypes.

- PD-L1 expression is a positive predictive factor of response.

- It is important to assess the level of expression of PD-L1 in TNBC in order to select patients who may benefit from available therapies.

Methods for assessing PD-L1 expression

- There are several PD-L1 assays for use with each anti-PD-L1 drug.

- The immunohistochemistry assay includes an antibody clone (22C3, 28-8, SP142, SP263 and 73-10) to be used with a specific detection platform (DAKO Autostainer Link 48 platform, Agilent Inc and VENTANA BenchMark Ultra platform, Ventana Medical Systems Inc.).

- The sensitivity of the detection kit for detecting PD-L1 varies depending on whether the test is performed on tumor cells (TCS) or immune cells (ICS).

- The SP142 kit stains more ICs but fewer TCs than kits based on the other PD-L1 antibodies. Conversely, the 22C3 and 28-8 antibodies show robust sensitivity with TCS, but less pronounced staining of ICs.

- The only PD-L1 assay licensed as a companion diagnostic device for selecting TNBC patients who are candidates for atezolizumab plus nab-paclitaxel is the VENTANA platform using the SP142 antibody clone.

Discussion

- The VENTANA PD-L1 (SP142) assay has proven to be highly sensitive for PD-L1 expression on ICs, but less sensitive for PD-L1 detection on TCs. However, scientific evidence has shown that this detection system identified patients treated with atezolizumab plus nab-paclitaxel with longer median progression-free survival and overall survival than the other platforms.

- Inter-reader agreement on PD-L1 IC positivity (0.805) was higher with the VENTANA PD-L1 (SP142) assay than with the VENTANA SP263 (0.616), DAKO 22C3 (0.605) and DAKO 28-8 (0.460) assays.

- According to the VENTANA PD-L1 (SP142) assay, the expression of PD-L1 in ICs is significantly higher in primary tumors than in metastatic tumors; in addition, expression is highest in the lymph nodes.

- Liver and bone samples should not be the first samples evaluated to assess PD-L1 expression.

- Expression of PD-L1 in lymph node metastases is more difficult to assess with the VENTANA PD-L1 (SP142) assay because this detection system considers only ICs, lymph nodes contain abundant ICs and it is difficult to delimit the tumor area in lymphoid tissue.

- PD-L1 status should be routinely assessed in the primary tumor, and, if the result is negative, the evaluation should be made in a recent metastatic sample.

- The pathology report for TNBC must include the antibody clone and the platform used for detection of PD-L1, as well as qualitative and quantitative expression data.

\section{References}

Papers of special note have been highlighted as: $\bullet$ of interest; $\bullet \bullet$ of considerable interest

1. Yu LY, Tang J, Zhang CM et al. New immunotherapy strategies in breast cancer. Int. J. Environ. Res. Public Health 14(1), 68 (2017).

2. Cimino-Mathews A, Foote JB, Emens LA. Immune targeting in breast cancer. Oncology (Williston Park) 29(5), 375-385 (2015).

3. Bertucci F, Goncalves A. Immunotherapy in breast cancer: the emerging role of PD-1 and PD-L1. Curr. Oncol. Rep. 19(10), 64 (2017).

4. Cao Y, Axup JY, Ma JS et al. Multiformat T-cell-engaging bispecific antibodies targeting human breast cancers. Angew. Chem. Int. Ed. Engl. 54(24), 7022-7027 (2015).

5. Berry DA, Cirrincione C, Henderson IC et al. Estrogen-receptor status and outcomes of modern chemotherapy for patients with node-positive breast cancer. JAMA 295(14), 1658-1667 (2006).

6. Den Brok WD, Speers CH, Gondara L, Baxter E, Tyldesley SK, Lohrisch CA. Survival with metastatic breast cancer based on initial presentation, de novo versus relapsed. Breast Cancer Res. Treat. 161(3), 549-556 (2017).

7. Bonotto M, Gerratana L, Poletto $\mathrm{E}$ et al. Measures of outcome in metastatic breast cancer: insights from a real-world scenario. Oncologist 19(6), 608-615 (2014).

8. NCCN Clinical Practice Guidelines in Oncology (NCCN Guidelines ${ }^{\circledR}$ ). Breast cancer version 4.2020. (2020). https://www.nccn.org/professionals/physician_gls/pdf/breast.pdf

9. Cardoso F, Senkus E, Costa A et al. 4th ESO-ESMO international consensus guidelines for advanced breast cancer (ABC 4). Ann. Oncol. 29(8), 1634-1657 (2018).

10. Ruffell B, Au A, Rugo HS, Esserman LJ, Hwang ES, Coussens LM. Leukocyte composition of human breast cancer. Proc. Natl Acad. Sci. USA 109(8), 2796-2801 (2012). 
11. Liu S, Lachapelle J, Leung S, Gao D, Foulkes WD, Nielsen TO. CD8+ lymphocyte infiltration is an independent favorable prognostic indicator in basal-like breast cancer. Breast Cancer Res. 14(2), R48 (2012).

12. Mittendorf EA, Philips AV, Meric-Bernstam F et al. PD-L1 expression in triple-negative breast cancer. Cancer Immunol. Res. 2(4), 361-370 (2014).

13. Katz H, Alsharedi M. Immunotherapy in triple-negative breast cancer. Med. Oncol. 35(1), 13 (2017).

14. Emens LA, Cruz C, Eder JP et al. Long-term clinical outcomes and biomarker analyses of atezolizumab therapy for patients with metastatic triple-negative breast cancer: a Phase I study. JAMA Oncol. 5(1), 74-82 (2019).

15. Emens LA, Adams S, Barrios CH et al. LBA16 IMpassion130: final OS analysis from the pivotal Phase III study of atezolizumab + nab-paclitaxel vs placebo + nab-paclitaxel in previously untreated locally advanced or metastatic triple-negative breast cancer. Ann. Oncol. 31(Suppl. 4), S1148 (2020).

-• Pivotal Phase III IMpassion130 trial that compared atezolizumab plus nab-paclitaxcel with placebo in patients with locally advanced or metastatic triple-negative breast cancer (TNBC) that have not received previous treatment.

16. Nanda R, Chow LQ, Dees EC et al. Pembrolizumab in patients with advanced triple-negative breast cancer: Phase Ib KEYNOTE-012 study. J. Clin. Oncol. 34(21), 2460-2467 (2016).

17. Cortes J, Cescon DW, Rugo HS et al. KEYNOTE-355: randomized, double-blind, Phase III study of pembrolizumab + chemotherapy versus placebo + chemotherapy for previously untreated locally recurrent inoperable or metastatic triple-negative breast cancer. J. Clin. Oncol. 38(Suppl. 15), Abstract 1000 (2020).

-. Phase III KEYNOTE-355 study that compared pembrolizumab plus chemotherapy with placebo in patients with locally recurrent inoperable or metastatic TNBC that have not received previous treatment.

18. Ratcliffe MJ, Sharpe A, Midha A et al. Agreement between programmed cell death ligand-1 diagnostic assays across multiple protein expression cutoffs in non-small-cell lung cancer. Clin. Cancer Res. 23(14), 3585-3591 (2017).

19. Schats KA, van Vre EA, Boeckx C et al. Optimal evaluation of programmed death ligand-1 on tumor cells versus immune cells requires different detection methods. Arch. Pathol. Lab. Med. 142(8), 982-991 (2018).

- Article that compared the commercially available and approved PD-L1 immunohistochemistry assays (22C3, 28-8, SP142, SP263), specifically identifying the changes in staining output created by altering the detection method.

20. Karnik T, Kimler BF, Fan F, Tawfik O. PD-L1 in breast cancer: comparative analysis of 3 different antibodies. Hum. Pathol. 72, 28-34 (2018).

21. Kintsler S, Cassataro MA, Drosch M, Holenya P, Knuechel R, Braunschweig T. Expression of programmed death ligand (PD-L1) in different tumors. Comparison of several current available antibody clones and antibody profiling. Ann. Diagn. Pathol. 41, 24-37 (2019).

22. Noske A, Amman J, Wagner DC et al. Reproducibility and concordance of 4 clinically developed programmed death-ligand 1 (PD-L1) immunohistochemistry (IHC) assays in triple negative breast cancer (TNBC). Ann. Oncol. 30(Suppl. 5), v130-131 (Abstract 359P) (2019).

- Poster presented at the European Society for Medical Oncology 2019 Congress that compares PD-L1-IC-positivity (as a percentage of the tumour area) across the four assays, adjusted for reader effects.

23. Ionescu DN, Downes MR, Christofides A, Tsao MS. Harmonization of PD-L1 testing in oncology: a Canadian pathology perspective. Curr. Oncol. 25(3), e209-e216 (2018).

24. Lantuejoul S, Sound-Tsao M, Cooper WA et al. PD-L1 testing for lung cancer in 2019: perspective from the IASLC Pathology Committee. J. Thorac. Oncol. 15(4), 499-519 (2020).

25. Parra ER, Villalobos P, Mino B, Rodriguez-Canales J. Comparison of different antibody clones for immunohistochemistry detection of programmed cell death ligand 1 (PD-L1) on non-small cell lung carcinoma. Appl. Immunohistochem. Mol. Morphol. 26(2), 83-93 (2018).

- Study conducted to compare and validate the available commercial PD-L1 clones and identify which ones can be reliably used by the surgical pathologist to evaluate the immunohistechemistry PD-L1 expression, using lung cancer as a model.

26. US FDA. FDA approves atezolizumab for first-line treatment of metastatic NSCLC with high PD-L1 expression (2020). https://www.fda.gov/drugs/resources-information-approved-drugs/fda-approves-atezolizumab-first-line-treatment-metastatic-nsclc-hig h-pd-11-expression

27. US FDA. List of cleared or approved companion diagnostic devices (in vitro and imaging tools) (2020). https: //www.fda.gov/medical-devices/vitro-diagnostics/list-cleared-or-approved-companion-diagnostic-devices-vitro-and-imaging-tools

28. US FDA. FDA approves atezolizumab for PD-L1 positive unresectable locally advanced or metastatic triple-negative breast cancer (2020). https://www.fda.gov/drugs/drug-approvals-and-databases/fda-approves-atezolizumab-pd-11-positive-unresectable-locally-adv anced-or-metastatic-triple-negative

29. Schmid P, Adams S, Rugo HS et al. Atezolizumab and nab-paclitaxel in advanced triple-negative breast cancer. N. Engl. J. Med. 379(22), 2108-2121 (2018).

30. Schmid P, Rugo HS, Adams S et al. Atezolizumab plus nab-paclitaxel as first-line treatment for unresectable, locally advanced or metastatic triple-negative breast cancer (IMpassion130): updated efficacy results from a randomised, double-blind, placebo-controlled, Phase 3 trial. Lancet Oncol. 21(1), 44-59 (2020). 
-• Updated efficacy results of the Phase III IMpassion130 study, that compared atezolizumab plus nab-paclitaxel with placebo in patients with unresectable, locally advanced or metastatic TNBC that have not received previous treatment.

31. Hirsch FR, Mcelhinny A, Stanforth D et al. PD-L1 immunohistochemistry assays for lung cancer: results from Phase 1 of the blueprint PD-L1 IHC assay comparison project. J. Thorac. Oncol. 12(2), 208-222 (2017).

32. Rugo HS, Loi S, Adams S et al. Performance of PD-L1 immunohistochemistry assays in unresectable locally advanced or metastatic triple-negative breast cancer: post hoc analysis of IMpassion130. Ann. Oncol. 30(Suppl. 5), v858-959 (Abstract LBA820) (2019).

-. Exploratory analysis of IMpassion130 substudy in which 22C3 and SP263 PD-L1 immunohistochemistry assays were evaluated for PD-L1 prevalence, analytical concordance with SP142 and estimates of clinical activity.

33. US FDA. Data sheet for VENTANA PD-L1 (SP142) Assay (2020). https://www.accessdata.fda.gov/cdrh_docs/pdf16/p160002s009c.pdf

34. Dennis E, Kockx MM, Harlow G, Cai Z, Bloom K, Elgabry E. Effective and globally reproducible digital pathologist training program on PD-L1 immunohistochemistry scoring on immune cells as a predictive biomarker for cancer immunotherapy in triple negative breast cancer. Cancer Res. 80(Suppl. 4), (Abstract PD5-02) (2020).

35. Reisenbichler ES, Han G, Bellizzi A et al. Prospective multi-institutional evaluation of pathologist assessment of PD-L1 assays for patient selection in triple negative breast cancer. Mod. Pathol. 33, 1746-1752 (2020).

36. Plasilova ML, Hayse B, Killelea BK, Horowitz NR, Chagpar AB, Lannin DR. Features of triple-negative breast cancer: analysis of 38,813 cases from the national cancer database. Medicine (Baltimore) 95(35), e4614 (2016).

37. Li Y, Vennapusa B, Chang CW et al. Prevalence study of PD-L1 SP142 assay in metastatic triple-negative breast cancer. Appl. Immunohistochem. Mol.Morphol. doi:10.1097/PAI.0000000000000857 (2020) (Epub ahead of print).

38. Exman P, Garrido-Castro A, Tolaney S. PD-L1 testing in patients with breast cancer: controversies and current practice. Curr. Breast Cancer Rep. 11, 353-357 (2019).

39. Pelekanou V, Barlow WE, Nahleh ZA et al. Tumor-infiltrating lymphocytes and PD-L1 expression in pre- and posttreatment breast cancers in the SWOG S0800 Phase II neoadjuvant chemotherapy trial. Mol. Cancer Ther. 17(6), 1324-1331 (2018).

40. Li X, Warren S, Pelekanou V et al. Immune profiling of pre- and post-treatment breast cancer tissues from the SWOG S0800 neoadjuvant trial. J. Immunother. Cancer 7(1), 88 (2019).

41. Dieci MV, Tsvetkova V, Griguolo G et al. Integration of tumour infiltrating lymphocytes, programmed cell-death ligand-1, CD8 and FOXP3 in prognostic models for triple-negative breast cancer: Analysis of 244 stage I-III patients treated with standard therapy. Eur. J. Cancer 136, 7-15 (2020).

42. Yuan C, Liu Z, Yu Q et al. Expression of PD-1/PD-L1 in primary breast tumours and metastatic axillary lymph nodes and its correlation with clinicopathological parameters. Sci. Rep. 9(1), 14356 (2019).

43. Schrijver WA, van der Groep P, Hoefnagel LD et al. Influence of decalcification procedures on immunohistochemistry and molecular pathology in breast cancer. Mod. Pathol. 29(12), 1460-1470 (2016).

44. Li Y, Chang CW, Tran D, Denker M, Hedge P, Molinero L. Prevalence of PDL1 and tumor infiltrating lymphocytes (TILs) in primary and metastatic TNBC. Cancer Res. 78(Suppl. 4), (Abstract PD6-01) (2018).

45. FDA summary of safety and effectiveness data for VENTANA PD-L1 (SP142) assay (2020). https://www.accessdata.fda.gov/cdrh_docs/pdf16/p160002s009b.pdf

46. Garrido P, Conde E, De Castro J et al. Updated guidelines for predictive biomarker testing in advanced non-small-cell lung cancer: a National Consensus of the Spanish Society of Pathology and the Spanish Society of Medical Oncology. Clin. Transl. Oncol. 22(7), 989-1003 (2020).

47. International Association for the Study of Lung Cancer. Soo RA, Asuncion BR, Buettner R. PD-L1 SP142 assay. IASLC Atlas of PD-L1 Immunohistochemistry Testing in Lung Cancer. Tsao MS, Kerr KM, Dacic S, Yatabe Y, Hirsch FR (Eds). Rx Press, CO, USA, 63-72 (2017). 